\title{
Towards on line monitoring the evolution of the myocardium infarction scar with an implantable electrical impedance spectrum monitoring system.
}

\author{
B Sanchez, A Guasch, P. Bogonez, C Galvez, V Puig, C Prat, C E Semino, A Bayes-Genis and R Bragos
}

\begin{abstract}
The human heart tissue has a limited capacity for regeneration. Tissue and cellular therapies based on the use of stem cells may be useful alternatives to limit the size of myocardial infarction. In this paper, the preliminary results from an experimental campaign for on-line monitoring of myocardium scar infarction are presented. This study has been carried out under a research project that has as main objective the development and application of a bioactive patch implant for regeneration of myocardial infarction. Electrical Impedance Spectroscopy (EIS) has been chosen as a tissue state monitoring technique. What is presented in this communication is the first results of an implantable EIS measurement system which has been implanted in a subset of the animals corresponding to the control group, along one month. In all the animals, the myocardial infarction was induced by the ligation of the first circumflex marginal artery. In the animal group presented, the bioactive patch scaffold and the electrodes were implanted without the stem cells load. The scaffold is a piece of decellularized human pericardium, lyophilized and rehydrated with hydrogel RAD16-I. Nanogold particles were also placed near the electrodes to improve the electrode area conductivity. The results presented correspond to the subset of animals $(n=5)$, which had implanted the bioimpedance system monitoring the electrical impedance spectrum in vivo during 1 month. Two electrodes were connected to the bioactive patch implant. A total of 14 logarithmically spaced frequencies were measured every 5 minutes, from $100 \mathrm{~Hz}$ to $200 \mathrm{kHz}$. Results show a convergence of low-frequency and high frequency impedance magnitudes along the measurement period, which is coherent with the scar formation.
\end{abstract}

\section{INTRODUCTION}

Heart failure is the end-stage of many cardiovascular diseases. One of the main problems is the presence of necrotic tissue due to an acute myocardial infarction which deeply affects heart function. Due to myocardium has very limited regeneration capacity and the number of transplant donors hardly meets the demand of potential recipients, new therapeutic approaches to recover this function are highly

This work has been supported in part by the Spanish Ministry MICINN SAF2008-05144-C02-02/01/03 and SAF2011-30067-C02-02/01, 080331/0/2 from Fundació La Marató de TV3, by the Redes de Investigacion del Instituto de Salud Carlos III (REDINSCOR, RD06/0003), Fondo Europeo de Desarrollo Regional (FEDER).

C Galvez-Monton, C Prat-Vidal, A Bayes-Genis are with ICREC Research Program, IGTP, Hospital Universitari Germans Trias i Pujol, Badalona, SPAIN.

V Puig and C E Semino are with the Tissue Engineering Laboratory, Bioengineering Department, Institut Quimic de Sarria, Universidad Ramon Llull, Barcelona, SPAIN.

B Sanchez, A Guasch, P. Bogonez and R Bragos are with the Departament d'Enginyeria Electronica, Universitat Politecnica de Catalunya (UPC), Barcelona, SPAIN. ramon.bragos @upc . edu required. Current treatments under development are looking for effective and less invasive treatments, hence therapies based on stem cells open a new world of possibilities since they can divide and differentiate into different specialized cell types.

One of the major concerns about tissue engineering for cardiac regeneration is to promote the regeneration or eventually replace necrotic tissue that has been lost or compromised i.e. due to an infarct, by a functional biological grafts. Ideally, an engineered graft should be able to recover, maintain, or even enhance the tissue functionality, being integrated with the host tissue in response to the native environmental factors [1]

Engineered tissues are usually developed in vitro for an in vivo implantation to direct remodeling and reparation/regeneration of tissues with the aim to enhance partial or total tissue function. Some authors show the beneficial effects of these platforms [2], [3]. However, controling the location of grafted cells after transplantation is difficult, since myocardium is a highly vascularized tissue. In general, there are two main approaches in tissue engineering field. The first consists in using isolated cells as replacement parts. The second, cell encapsulation into natural or artificial biomaterials (collagens, polymeric fibers, respectively) capable of inducing tissue regeneration, which could be a better option to maintain the cells in the infarcted zone [4].

The final aim of the research project is the development of an implanable bioactive patch to regenerate myocardium tissue. The development and results presented in this manuscript are focused only in a particular aspect, which is the on-line monitor of the tissue state in a nondestructive way by incorporating the sensing electrodes to the biocompatible membrane. The ultimate goal is to evaluate the beneficial effect of the membrane developed ad hoc in a porcine model of myocardial infarction monitoring the myocardial viability evolution through an implantable electronic system.

\section{SYSTEM DESCRIPTION}

The prototype used for the experimental measurements was originally presented in [5] where the system and characterization results were described. Five improved replicas were built for the measurement campaign. The bioimpedance measurement system is a custom implantable bioimpedance meter with a wireless Zigbee link. A block diagram is show in Figure 1. The main parts are: (1) a device that enables 
the measurement of tissue impedance, (2) a wireless link to transmit impedance data and (3) a computer to log, process and display data. The bioimpedance measuring device is based on the AD5933 from Analog Devices and the MSP430 microcontroller from Texas Instruments. The system enables measurements at 4 and 2 electrodes every 5 minutes (programmable) for 14 different frequencies selectable between $100 \mathrm{~Hz}$ and $200 \mathrm{kHz}$. The measured impedance range is between $10 \Omega$ and $10 \mathrm{k} \Omega$. An analog front end has been implemented to enable the measurement of low impedances trough high electrode impedances. The front end consists in a voltage controlled current source with a bandwidth of $1 \mathrm{MHz}$ to inject an ac current to the tissue under measurement, which is controlled by the AD5933 signal generator and is limited to $10 \mu A_{p p}$. A wide-bandwidth differential voltage amplifier measures the voltage drop between electrodes. The system monitors the real an imaginary parts of the impedance under test, the battery level and the internal temperature. All these data are sent to the computer through the Zigbee wireless network and are also stored into a flash memory which content can be uploaded through the Zigbee link in situ or after extracting the device from the animal. The electrodes are built from a modified DSI Coupler Lead, Data Science International, by leaving the distal end $(5 \mathrm{~mm})$ uncoated and with only one of the two imbricated spirals. This allows the easy and robust connection of the spiral winding to the pericardial piece and also its sterilization by gamma radiation. In the subsequent hydration of the pericardium, when the hydrogel is formed, the mechanical properties of the set are maintained.

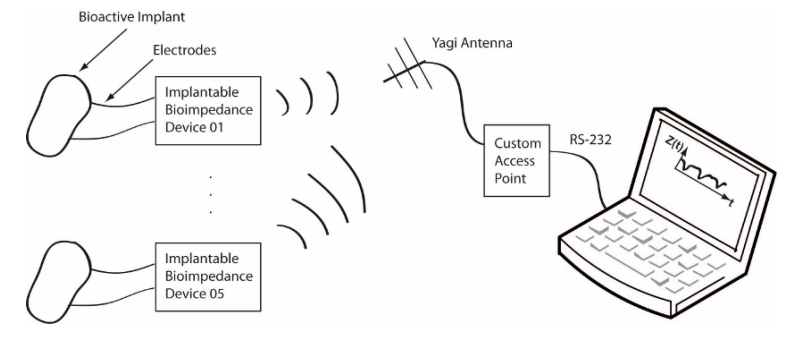

Fig. 1. Draft of the implemented system. Several bioimpedance monitors are implanted and connected to the receiver Access Point. See text for details.

The implantable system is powered by a Li-ion battery $(3.7 \mathrm{~V})$ with $1000 \mathrm{mAh}$ capacity. The digital part of the system is powered at 3.3 volts whereas the analog subcircuits and the wireless device are powered at \pm 2.8 volts. The microcontroller is in charge on managing the powerup and power-down of analog and RF parts of the system in order to reduce the global system consumption. The data packet sent is as follows: one byte for the device identifier (to enable multiple device monitoring), one byte for the Receiver Signal Strength Indication (RSSI) value, one byte for the measured frequency and two bytes for each the real and the imaginary parts. Then the temperature and the battery voltage respectively (11 Bytes each). A LabVIEW program has been implemented to receive the data in a computer. The program provides a serial communication with the access point, receives and processes the data and stores them in a file.

\section{MethodS}

\section{A. Scaffold}

The structure of the bioactive patch is formed by a piece of decellularized human pericardium by treatment with detergents. The metallic electrodes are anchored to the pericardium before the sterilization and hydratation. The electrical connection with the matrix is improved by permeating the region with a solution of nanogold to favor the flow of electrical current within the biological membrane to the peptide hydrogel. This solution is only applied to the area where the electrodes are nested in the pericardial piece.

RAD16-I peptide hydrogel is used to fill in the decellularized human pericardium scaffold. RAD16-I is commercially available under the name of PuramatrixTM (BD, Erembodegem, Belgium). This peptide is an ideal candidate as cell culture matrix in tissue engineering since its biomechanical properties are similar to natural extracellular matrices [6]. It provides a convenient mechanism for self-assembly into highly organized nanostructures associated with 3D network formation, which may promote cell adhesion, spreading, migration, growth and differentiation. Extensive studies have already indicated their good biocompatibility and biodegradability [7].

\section{B. Bioimpedance monitor encapsulation}

The bioimpedance monitors $(60 \mathrm{mmx} 45 \mathrm{mmx} 24 \mathrm{~mm})$ were covered with a biocompatible polydimethylsiloxane silicone (PDMS) (Sylgard, 184, Dow Corning Corp.). The PDMS was prepared by mixing two liquid components in a ratio of 10 parts base to one part curing agent in a liquid state $(10: 1$, by weight) (Sylgard, 184, Dow Corning Corp.). Air introduced into the mixture during stirring was removed by using a vacuum chamber at 700 mbar for 30 minutes. Then, the mixture was smoothly poured over the bioimpedance system box. To shorten the curing time, the mixture was cured in an oven (60 Celsius degrees, 2.5-3h). The implantable systems were sterilised by prior to the implantation using chemical agents.

\section{Animal preparation}

Human pericardium was obtained from patients undergoing cardiac surgery. This protocol was approved by the local Ethics Committee, and informed consent was obtained from all patients. The study protocol conformed to the principles outlined in the Declaration of Helsinki.

A total of 5 Pigs $(20-30 \mathrm{~kg})$ were premedicated with an IM mixture of ketamine $(20 \mathrm{mg} / \mathrm{kg})$, diazepam $(0.25 \mathrm{mg} / \mathrm{kg})$, and atropine $(0.25 \mathrm{mg} / \mathrm{kg})$. Anesthetic induction was done with an IV propofol bolus ( $4 \mathrm{mg} / \mathrm{kg}$ ). Animals underwent endotracheal intubation, and anesthesia was maintained by $2 \%$ sevofluorane inhalation. A continued IV infusion of Ketorolac $(0.15 \mathrm{mg} / \mathrm{kg} / \mathrm{h})$ and tramadol $(0.5 \mathrm{mg} / \mathrm{kg} / \mathrm{h})$ was used 
as an intraoperatory analgesic. At the beginning of the intervention, an IV lidocaine bolus $(1 \mathrm{mg} / \mathrm{kg})$ was administered, followed by a continuous infusion $(1 \mathrm{mg} / \mathrm{kg} / \mathrm{h})$ maintained for $1 \mathrm{~h}$ after myocardial infarction (MI) induction. At the end of the surgery, $2.5 \mathrm{mg} / \mathrm{kg}$ of tulatromicin, IM (Draxxin reg., Pfizer) was administered as antibiotic therapy.

Acute MI was induced by a double-ligation (Prolene 5/0) of the first marginal branch of the circumflex coronary artery, $1.5 \mathrm{~cm}$ distal from the atrioventricular groove. 30 minutes after MI induction, the bioactive patch connected to the bioimpedance monitoring system was implanted with surgical glue (Glubran2) covering the infarcted area. All procedures were done under monitoring conditions with ECG registration and measures of capnography, pulse oximetry, non-invasive arterial blood pressure and temperature. Animals underwent magnetic resonance imaging (MRI) at multiple time points (baseline, $2 \mathrm{~h}$ and 1 month after MI) to assess functional recovery. All pigs were sacrificed at 1 month to obtain samples for the histological analysis.

\section{EXPERIMENTAL RESULTS}

A total of 7 animals comprised the control group. In all animals myocardial infarction was induced by ligation of the first circumflex marginal artery. In the control group the membrane (decellularized pericardium, lyophilized and rehydrated with nanogold and hydrogel) was implanted. In a subset of 5 animals, five replicas of the bioimpedance monitor were implanted and connected to the electrode cables which were attached to the bioactive patch to monitor tissue EIS in vivo. The performance of the network formed by the telemetry equipment and the external implantable receiver advised to reduce to 5 the number of systems in simultaneous operation. During the course of the experimental measurements, we have collaborated with the Minimally Invasive Surgery Center Jesus Uson (CCMIJU) in Caceres, Spain, where the model of acute MI in pigs and the measurement of cardiac function by magnetic resonance imaging was performed.

\section{A. Implantation of the bioimpedance monitoring system}

Figure 2 shows a detail of the bioactive patch with the electrodes and the first section of cable, which is sterilized by gamma radiation, and the process of implementing the patch, the connection of cables with the implantable system, which has been introduced through an auxiliary access practiced in the animal shoulder.

Next, Figure 3 shows the implantable system closed and sealed with biocompatible silicone (Sylgard 184) as specified in Section III as well as the measurement setup at the animal care center. Furthermore, it was possible to remotely monitorize the evolution of the MI ischemia just using the remote desktop. The goal of this feature was to detect anomalies in the measurements, for example, due to poor electrode contact in the bioactive patch implant, but the batch upload of the full memory content of each device was used in practice.

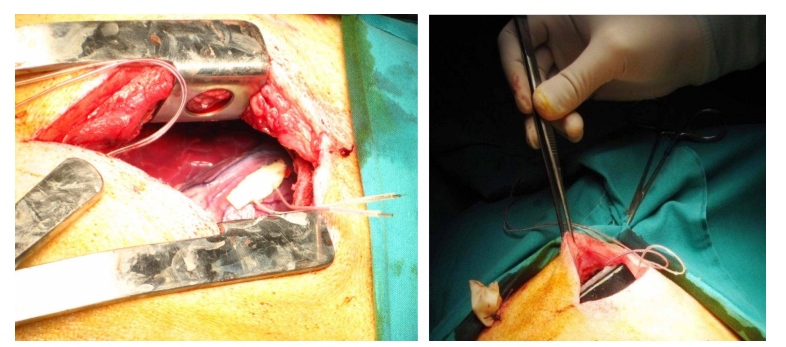

Fig. 2. Detail of the implant with the electrodes and the first section of cable, which is sterilized by gamma radiation (left). Subcutaneous location of the bioimpedance implanted system (right).

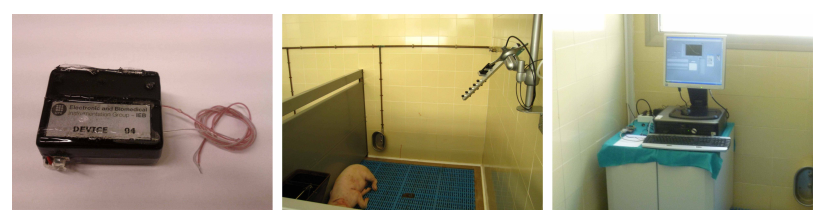

Fig. 3. From left to right: implantable system sealed with biocompatible silicone. Telemetry system in the animal care center: the receiving antenna was oriented toward animals monitored to improve the quality of data transmission. The antenna was connected to the receiving system using a coaxial cable. Through the labview management program, the received data were stored in text files. Magnitude and phase angle of impedance data were also displayed for each animal being measured $(n=5)$. From the systems implanted, we could retrieved measures from 4 of them (one of them was destroyed by the animal).

\section{B. Long-term monitoring of myocardial infarct scar}

The estimates on the state of the tissue that may arise, according to the literature and in vivo measurements obtained by the group in previous projects, derived from measurements of magnitude and phase angle at low (around $10 \mathrm{kHz}$ ) and high (around $200 \mathrm{kHz}$ ) frequencies, and variations on the ratio between them. Following Figure 4 shows the evolution of the impedance magnitude at three consecutive frequencies measured in a control animal. To filter the noise and artifacts into the measurements, a median and a moving average filter was used at each frequency and the measurement at low and high frequency measurements were obtained by averaging measurements at three frequencies in each range.

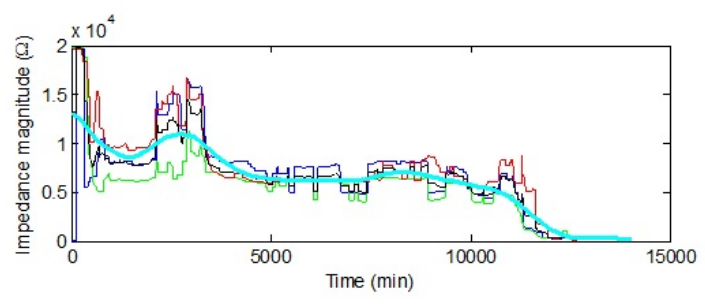

Fig. 4. Evolution of the impedance magnitude at low frequency in a control animal. Three consecutive frequencies are represented.

Figure 5 shows the evolution of the impedance magnitude at low (around $10 \mathrm{kHz}$ ) and high (around $100 \mathrm{kHz}$ ) measured in two different animals from the control group. A systematic increase of the initial magnitude can be observed. This is coherent with the previously described impedance increase in the acute ischemia [8] but can also be due to the patch 
and electrodes integration with the tissue. Then comes a maintenance phase. There is a period with greater separation between low and high frequencies which could corresponds to the lysis of cells and scar formation finishing between 4,5 and 7 days after infarction. Finally, the stationary phase where both low and high frequency impedance magnitude have the same value, which is coherent with the previously described resistive behaviour of the myocardium scar. The final drop in the impedance corresponds to the depletion of the battery, which happened before the calculated 1 month period in all systems, ranging from 10 to 25 days.
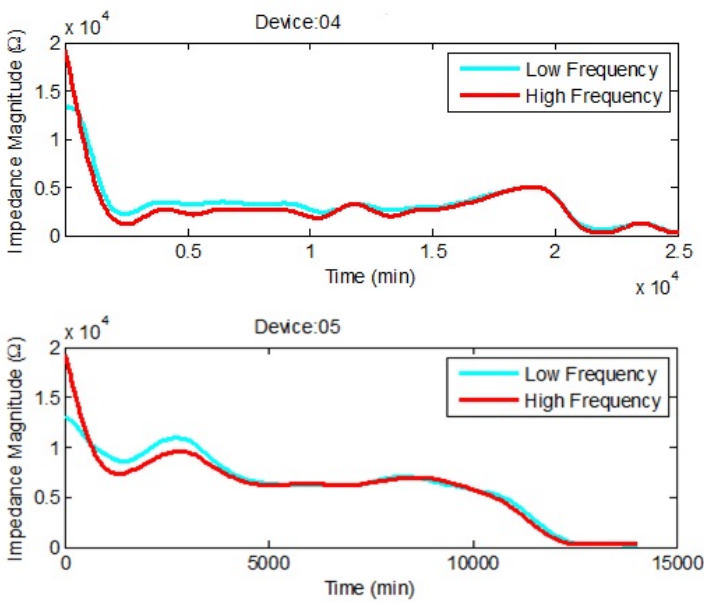

Fig. 5. Evolution of the impedance magnitude at low and high frequency in two control animals.

\section{Discussion}

Although previous validation carried out with the first built unit, we have found problems that limited their network operation in long periods. In practice, the coexistence of the five systems and the actual attenuation of the telemetry signal gave error rates higher than those found with a single unit, described in [5]. Both the embedded microcontroller and computer control software were redesigned to provide higher performance and system management and retrieval capabilities of the harvested data.

Although these results are preliminary, we must emphasize that, to the best of our knowledge, there are no previous descriptions in the literature on measurements of these characteristics. The development of an implantable EIS monitoring system that incorporates miniaturized telemetry has enabled continuous EIS measurements at 14 frequencies every 5 minutes during 1 month in pigs. The results are coherent with the already known behavior of the three tissue states: normal, acute ischemia and healed scar and provide clues about the dynamics of the transition between these states.

\section{ACKNOWLEDGEMENTS}

The authors would like to thank Dr. Idoia Diaz-Guemes from the Minimally Invasive Surgery Center Jesus Uson at Caceres (Spain), for her contribution to the animal experimentation.

\section{REFERENCES}

[1] G. Vunjak-Novakovic, N. Tandon, A. Godier, R. Maidhof, A. Marsano, T. Martens, and M. Radisic, "Challenges in cardiac tissue engineering." Tissue engineering. Part B, Reviews, vol. 16, no. 2, pp. 169-87, Apr. 2010.

[2] J. D. Dowell, M. Rubart, K. B. S. Pasumarthi, M. H. Soonpaa, and L. J. Field, "Myocyte and myogenic stem cell transplantation in the heart." Cardiovascular research, vol. 58, no. 2, pp. 336-50, May 2003.

[3] A. Bayes-Genis, C. Soler-Botija, J. Farré, P. Sepúlveda, A. Raya, S. Roura, C. Prat-Vidal, C. Gálvez-Montón, J. A. Montero, D. Büscher, and J. C. Izpisúa Belmonte, "Human progenitor cells derived from cardiac adipose tissue ameliorate myocardial infarction in rodents." Journal of molecular and cellular cardiology, vol. 49, no. 5, pp. 77180, Nov. 2010.

[4] A. Khademhosseini, R. Langer, J. Borenstein, and J. P. Vacanti, "Microscale technologies for tissue engineering and biology." Proceedings of the National Academy of Sciences of the United States of America, vol. 103, no. 8, pp. 2480-7, Feb. 2006.

[5] P. Bogonez-Franco, R. Bragos, A. Bayes-Genis, and J. Rosell-Ferrer, "Implantable bioimpedance monitor using ZigBee." IEEE Conference of the Engineering in Medicine and Biology Society (EMBS)., pp. 486871, Jan. 2009.

[6] C. E. Semino, "Can We Build Artificial Stem Cell Compartments?" Journal of biomedicine \& biotechnology, no. 3, pp. 164-169, Jan. 2003.

[7] — "Self-assembling Peptides: From Bio-inspired Materials to Bone Regeneration," Journal of Dental Research, vol. 87, no. 7, pp. 606-616, Jul. 2008.

[8] Y. Salazar, R. Bragos, O. Casas, J. Cinca, and J. Rosell, "Transmural versus nontransmural in situ electrical impedance spectrum for healthy, ischemic, and healed myocardium." IEEE transactions on bio-medical engineering, vol. 51, no. 8, pp. 1421-7, Aug. 2004. 